\title{
The kinetic model of the solar wind influenced by the given magnetic field
}

\author{
Natalia R. Minkova ${ }^{1}$ \\ ${ }^{1}$ Department of Mathematical Physics, Tomsk State University, FTF, Lenin Avenue, 36, \\ Tomsk, 634050, Russia \\ email: nminkova@ftf.tsu.ru
}

\begin{abstract}
The solar wind is considered as a steady fully ionized hydrogen plasma flow, with rotational symmetry. The Parker-spiral type magnetic field specifies the dependence of the flow speed on the radial distance and meridional angle if the plasma is assumed to be quasi-neutral and currentless. A two-particle kinetic model of the collisionless rotationally symmetrical plasma flow in a magnetic field is formulated and applied to estimate the flux and density of the solar wind. The obtained theoretical results are compared to the observational data.
\end{abstract}

Keywords. solar wind, plasmas, magnetic fields, analytical, statistical

The kinetic model of the solar wind based on the Vlasov equations describes the plasma flow in terms of particle velocity distribution functions and a self-consistent electromagnetic field. This nonlinear model can be approximately investigated under some reasonable assumptions about the flow geometry and magnetic field (e.g. Parker 1958 and Pierrard, Issautier, Meyer-Vernet, et al. 2001). In this paper, the assumptions that specified the Parker magnetic field are modified allowing us to obtain the velocity profile of the solar wind. The two-particle kinetic approach to modeling a spherically symmetrical plasma flow in a negligible magnetic field (Vasenin \& Minkova 2003) is reformulated for the considered problem and used to estimate the solar wind flux and density.

The solar wind is considered as a steady rotationally symmetric flow of collisionless fully ionized hydrogen plasma. The approximation of quasi-neutral current-free plasma allows us to apply the two-particle kinetic model of dynamic electron-proton pairs (Vasenin \& Minkova 2003). The collisionless kinetic equation yields a general solutions for the two-particle velocity distribution function $f=f\left(E_{e}+E_{p}, M_{e}, M_{p}, \mu_{e}, \mu_{p}\right)$ in terms of an arbitrary function of the first integrals corresponding to the conservation laws for the total energy of electron and proton, $E_{e}+E_{p} \equiv m_{e} u_{e}^{2} / 2+m_{p} u_{p}^{2} / 2+m \varphi-m u_{\Omega}^{2} / 2=$ const, their angular momenta $M_{j} \equiv r \sin \theta\left(u_{j \phi}+u_{\Omega}+q_{j} A_{\phi} / c m_{j}\right)=$ const and magnetic moments $\mu_{j} \equiv c m_{j} v_{j}^{2} / q_{j} B \cong$ const in the co-rotating reference frame. Here, $u_{\Omega}=\Omega r \sin \theta, m=$ $m_{e}+m_{p} ; r, \theta, \phi$ are the spherical coordinates; $u_{j}\left(u_{j \phi}\right)$ is the magnitude of the particle vector velocity (the azimuthal component); $v_{j}$ is the Larmor-rotation velocity; $\varphi$ is the gravitational potential of the Sun; $\Omega$ is its angular rotation rate; $A_{\phi}$ is the azimuthal component of the vector potential $\mathbf{A}$ of magnetic field $(\mathbf{B}=\mu \mathbf{H}=\nabla \times \mathbf{A}, \mu=1)$. Index $\mathrm{j}$ refers to electrons (e) and protons (p). The assumed Maxwellian velocity distribution at the solar corona exobase, located at $r=r_{0}$, specifies the form of function $f$.

The Parker-type magnetic field is defined by assuming the frozen-in condition and specifying the azimuthal velocity, $U_{\phi}=\left(r-r_{0}\right) \Omega \sin \theta$ (in contrast to Parker's approach, the plasma outflow direction at $r=r_{0}$ is not prescribed). In this case the Maxwell equations yield the relation for the radial velocity with an integration constant $(\alpha)$ :

$$
U_{r}(r, \theta)=\alpha\left|B_{r 0}(\theta)\right| \sin ^{2} \theta\left(1-r / r_{0}\right)
$$



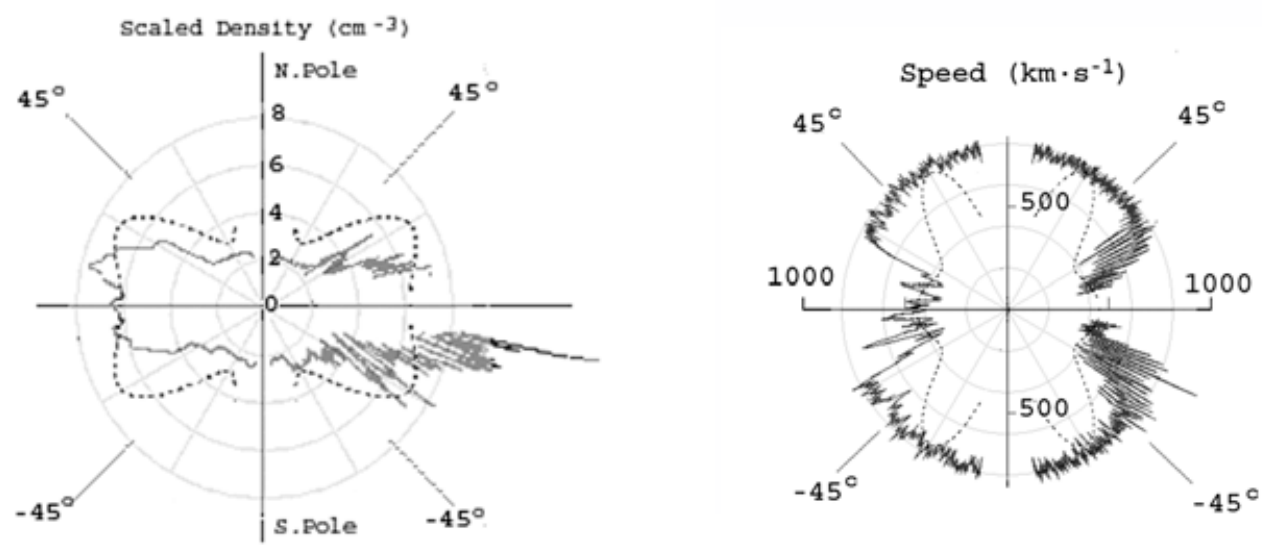

Figure 1. Ulysses data (solid) and model results (dotted) for solar wind density and speed.

under the assumption that there are no radial and meridional electric currents (a completely current-free flow is followed by $B_{r 0}=$ const). Equation (1.1), with $\alpha\left|B_{r 0}(\theta)\right|=$ $\alpha_{0}\left(1+16\left|\cos ^{7} \theta\right|\right)$ approximated according to the WSO magnetic field synoptic data (Wang \& Sheeley 1995), reproduces the profile similar to Ulysses "butterfly" diagram.

The analysis shows that the charge-particle drift cannot be neglected at high and low helio-latitudes for the present model. Hence these regions are not considered. The drift is also significant at $r \sim r_{0}$, increasing deviation of the model results from the observational data at the exobase. The drift equation yields the relation for the temperatures at $r=r_{0}$ :

$$
T_{e \perp 0}+T_{p \perp 0}=\frac{m\left|\varphi_{0}\right|}{6 k} \cdot \frac{1-u_{\Omega 0}^{2} /\left|\varphi_{0}\right|}{1-0.5\left(B_{\phi 0} / B_{0}\right)^{2}}
$$

that agrees for isotropic temperatures $\left(T_{0} \approx m\left|\varphi_{0}\right| / 8 k\right)$ with the observational coronal data for the slow and fast solar wind (Koehnlein 1996, Fisher \& Guhathakurta 1995).

The flux of plasma particles $F$ is evaluated from the distribution function $f$ for the nonmonotonic electron-proton potential $\Pi=m\left(\varphi-u_{\Omega}^{2} / 2\right)$. The model profiles of the plasma density $N=F / U_{r}$ and its speed $U_{r}$ (1.1) agree with the observational data (Koehnlein 1996) for the slow solar wind at $r \geqslant 1.5 r_{0}$ within $35 \%$. The meridional profiles of the density and the radial speed at $1 \mathrm{AU}$ (with $U_{r}=7.5 \cdot 10^{5} \mathrm{~m} / \mathrm{s}, N=3.1 \cdot 10^{6} \mathrm{~m}^{-3}$ for the latitude $60^{\circ}$ ) are qualitatively consistent with the corresponding Ulysses data (Fig.1). The parameter values are following: $r_{0}=3 R_{\odot}, T_{0}=9.6 \cdot 10^{5} \mathrm{~K}$ according to (1.2). The empirical terminal value of the in-ecliptic radial speed, $4.5 \cdot 10^{5} \mathrm{~m} / \mathrm{s}$ (Koehnlein 1996), is used for estimating $\alpha_{0}$. The parameter $N_{0}$ is found from the density measured at $1 \mathrm{AU}$.

Thus the suggested kinetic model describes the solar wind in the Parker-type magnetic field in terms of the electron-proton pairs statistics. This approach reproduces the radialangular profiles of the solar plasma density and speed consistent with the observations.

It is a pleasure to thank Prof. Y.M.Vasenin for initiating this research and discussions.

\section{References}

Parker E.N. 1958, ApJ 128, 664

Pierrard, V., Issautier, K., Meyer-Vernet N. \& Lemaire J. 2001, Geophys. Res. Let. 28, 2, 223

Vasenin, Y.M. \& Minkova, N.R. 2003, J. of Physics A. Mathematical and General, 36, 22, 6215

Wang, Y.-M. \& Sheeley, N. R., Jr. 1995, ApJ 447, L143

Koehnlein 1996, Solar Physics 169, 209

Fisher, R. \& Guhathakurta, M. 1995, ApJ 447, L139 